\title{
Nonnegative Definite EAP and ODF Estimation via a Unified Multi-shell HARDI Reconstruction
}

\author{
Jian Cheng ${ }^{1,2}$, Tianzi Jiang ${ }^{1}$, and Rachid Deriche ${ }^{2}$ \\ ${ }^{1}$ CCM, LIAMA, Institute of Automation, Chinese Academy of Sciences, China \\ 2 Athena Project Team, INRIA Sophia Antipolis - Méditerranée, France \\ jian.cheng.1983@gmail.com
}

\begin{abstract}
In High Angular Resolution Diffusion Imaging (HARDI), Orientation Distribution Function (ODF) and Ensemble Average Propagator (EAP) are two important Probability Density Functions (PDFs) which reflect the water diffusion and fiber orientations. Spherical Polar Fourier Imaging (SPFI) is a recent modelfree multi-shell HARDI method which estimates both EAP and ODF from the diffusion signals with multiple $b$ values. As physical PDFs, ODFs and EAPs are nonnegative definite respectively in their domains $\mathbb{S}^{2}$ and $\mathbb{R}^{3}$. However, existing ODF/EAP estimation methods like SPFI seldom consider this natural constraint. Although some works considered the nonnegative constraint on the given discrete samples of $\mathrm{ODF} / \mathrm{EAP}$, the estimated $\mathrm{ODF} / \mathrm{EAP}$ is not guaranteed to be nonnegative definite in the whole continuous domain. The Riemannian framework for ODFs and EAPs has been proposed via the square root parameterization based on pre-estimated ODFs and EAPs by other methods like SPFI. However, there is no work on how to estimate the square root of ODF/EAP called as the wavefuntion directly from diffusion signals. In this paper, based on the Riemannian framework for ODFs/EAPs and Spherical Polar Fourier (SPF) basis representation, we propose a unified model-free multi-shell HARDI method, named as Square Root Parameterized Estimation (SRPE), to simultaneously estimate both the wavefunction of EAPs and the nonnegative definite ODFs and EAPs from diffusion signals. The experiments on synthetic data and real data showed SRPE is more robust to noise and has better EAP reconstruction than SPFI, especially for EAP profiles at large radius.
\end{abstract}

\section{Introduction}

Diffusion MRI (dMRI) is the unique technique to explore the complex microstructure of white matter non-invasively, by modelling the diffusion of water molecules. The water diffusion is fully characterized by the diffusion Probability Density Function (PDF) called as the Ensemble Average Propagator (EAP). Under the narrow pulse assumption, the signal attenuation $E(\mathbf{q})$ is the Fourier transform of the EAP denoted by $P(\mathbf{R})[2]$ :

$$
E(\mathbf{q})=\int_{\mathbb{R}^{3}} P(\mathbf{R}) \exp \left(-2 \pi i \mathbf{q}^{T} \mathbf{R}\right) \mathrm{d} \mathbf{R}
$$

where $\mathbf{q}=q \mathbf{u}$ is the wavevector in imaging $\mathbf{q}$-space, and $\mathbf{R}=R \mathbf{r}$ is the displacement vector in spatial $\mathbf{R}$-space, and $\mathbf{u}$ and $\mathbf{r}$ are unit vectors. Since Diffusion Tensor Imaging (DTI) cannot handle the complex fiber configuration, a category of reconstruction

N. Ayache et al. (Eds.): MICCAI 2012, Part II, LNCS 7511, pp. 313-321, 2012.

(C) Springer-Verlag Berlin Heidelberg 2012 
methods, named as High Angular Resolution Diffusion Imaging (HARDI), were proposed to avoid the Gaussian EAP assumption in DTI [117|8 1|5|10]. In HARDI, EAP and two kinds of the Orientation Distribution Functions (ODFs) defined as $\Phi_{0}(\mathbf{r})=$ $\frac{1}{Z} \int_{0}^{\infty} P(R \mathbf{r}) \mathrm{d} R, \Phi_{2}(\mathbf{r})=\int_{0}^{\infty} P(R \mathbf{r}) R^{2} \mathrm{~d} R$, are normally used to infer fiber directions, where $Z$ in $\Phi_{0}(\mathbf{r})$ is the normalization factor to make $\Phi_{0}(\mathbf{r})$ as a PDF.

Spherical Polar Fourier Imaging (SPFI) is a recent multi-shell HARDI method, which represents the signal by Spherical Polar Fourier (SPF) basis [1] and analytically obtains EAP via the Fourier dual SPF basis [5] and the ODFs via Spherical Harmonic (SH) basis [3]. Although SPFI works well for the data with low SNR and non-exponential decay [3]5], the estimated ODF/EAP may have negative values. As physical PDFs, EAPs and ODFs should be nonnegative definite in $\mathbb{R}^{3}$ and $\mathbb{S}^{2}$ respectively. However, to our knowledge the existing ODF/EAP estimation methods like the classical Q-ball Imaging (QBI) [11]7] and the recent SPFI [15] seldom consider this constraint. Some works considered this constraint only on the given discrete PDF samples in $\mathbb{S}^{2}$ for ODFs [8] and in $\mathbb{R}^{3}$ for EAPs [10]. However, the discrete constraint only can ensure the estimated ODF/EAP is nonnegative on the given samples, while it may be negative in other samples. Moreover it is impractical for EAPs to consider the constraint on exhaustive samples in unbounded $\mathbb{R}^{3}[10]$. To our knowledge, there is still no work to estimate nonnegative definite ODFs/EAPs in the whole continuous domains.

The Riemannian framework has been proposed for tensor processing, e.g. the positive definite tensor estimation [9]. Recently the Riemannian framework has been generalized to ODF and EAP processing [4]6] by representing the square root of ODF/EAP called as the wavefunction with some orthonormal bases, and the wavefunction is calculated from the pre-estimated ODF/EAP by other methods like SPFI [15]. However since SPFI with the least square estimation does not consider the nonnegative constraint [15], the negative values of pre-estimated ODF/EAP must be forced to zero for the wavefunction estimation [6], which results in some numerical errors.

In this paper, we propose a model-free multi-shell HARDI method, named as Square Root Parameterized Estimation (SRPE), to estimate simultaneously the wavevector of EAP denoted as $\psi(\mathbf{R})$, the nonnegative EAP and ODFs from the diffusion signal samples. SRPE naturally guarantees the estimated ODF/EAP nonnegative definite in the continuous domain, not just in some discrete samples in [810]. The wavefunction estimated by SRPE can be used in the Riemannian framework without the numerical error introduced by negative values. Compared to SPFI, the experiments demonstrate the EAPs obtained in SRPE is more robust to noise especially at large radius.

\section{Square Root Parameterized Estimation (SRPE)}

\subsection{Analytical Relation between the Wavefunction $\psi(\mathrm{R})$ and the Signal $E(q)$}

SPF basis is a complete orthonormal basis which can sparsely represent Gaussian-like function with the first several basis functions [15], Motivated by the square root parameterization used in the Riemannian framework [4]6], we represent the wavefunction of EAP $P(\mathbf{R})$ as a linear combination of SPF basis in (2), where $G_{n}(R \mid \zeta) Y_{l}^{m}(\mathbf{r})$ is the SPF basis with the Gaussian-Laguerre function $G_{n}(R \mid \zeta)$ in radial part and the 
Spherical Harmonic (SH) basis $Y_{l}^{m}(\mathbf{u})$ in spherical part [15], and the coefficient vector $\boldsymbol{c}=\left(c_{000}, \ldots, c_{N L L}\right)^{T}$ has unit norm because $\int_{\mathbb{R}^{3}} P(\mathbf{R}) \mathrm{d} \mathbf{R}=1[6]$.

$$
\begin{array}{cc}
\psi(\mathbf{R})=\sum_{n=0}^{N} \sum_{l=0}^{L} \sum_{m=-l}^{l} c_{n l m} G_{n}(R \mid \zeta) Y_{l}^{m}(\mathbf{r}), & \|\boldsymbol{c}\|=1, \quad P(\mathbf{R})=(\psi(\mathbf{R}))^{2} \\
G_{n}(R \mid \zeta)=\kappa_{n}(\zeta) \exp \left(-\frac{R^{2}}{2 \zeta}\right) L_{n}^{1 / 2}\left(\frac{R^{2}}{\zeta}\right), & \kappa_{n}(\zeta)=\left[\frac{2}{\zeta^{3 / 2}} \frac{n !}{\Gamma(n+3 / 2)}\right]^{1 / 2}
\end{array}
$$

How to set the scale $\zeta$ will be discussed in 2.4. Please note that [6] represented $\psi(\mathbf{R})$ with Fourier dual SPF basis, while we use SPF basis. Actually different basis obtains the equivalent Riemannian framework as demonstrated in [6], however it is convenient for the analytical ODF and EAP estimation in 2.3 if $\psi(\mathbf{r})$ is represented by SPF basis.

By substituting the plane wave equation in (4) [5] into (1), where $j_{\alpha}(x)$ is the $\alpha$-th order spherical Bessel function, the signal $E(\mathbf{q})$ in (1) can be written as a function with respect to $c$ in (5), where the Fourier integration in $\mathbb{R}^{3}$ is separated into radial integration $I_{n n^{\prime} \alpha}(q)$ and spherical integration $Q_{l l^{\prime} \alpha}^{m m^{\prime} \beta}$ in (7).

$$
\begin{gathered}
e^{-2 \pi i \mathbf{q} \cdot \mathbf{R}}=4 \pi \sum_{\alpha=0}^{\infty} \sum_{\beta=-\alpha}^{\alpha}(-1)^{\alpha / 2} j_{\alpha}(2 \pi q R) Y_{\alpha}^{\beta}(\mathbf{u}) Y_{\alpha}^{\beta}(\mathbf{r}) \\
E(\mathbf{q} \mid \boldsymbol{c})=\int_{\mathbb{R}^{3}}\left(\sum_{n=0}^{N} \sum_{l=0}^{L} \sum_{m=-l}^{l} c_{n l m} G_{n}(R \mid \zeta) Y_{l}^{m}(\mathbf{r})\right)^{2} e^{-2 \pi i \mathbf{q}^{T} \mathbf{R}} \mathrm{d} \mathbf{R} \\
=\sum_{n l m} \sum_{n^{\prime} l^{\prime} m^{\prime}} \sum_{\alpha \beta} 4 \pi(-1)^{\frac{\alpha}{2}} c_{n l m} c_{n^{\prime} l^{\prime} m^{\prime}} I_{n n^{\prime} \alpha}(q \mid \zeta) Q_{l l^{\prime} \alpha}^{m m^{\prime} \beta} Y_{\alpha}^{\beta}(\mathbf{u})=\boldsymbol{c}^{T} \mathbf{K}(\mathbf{q} \mid \zeta) \boldsymbol{c} \\
\mathbf{K}_{n l m}^{n^{\prime} l^{\prime} m^{\prime}}(\mathbf{q} \mid \zeta)=\sum_{\alpha=0}^{2 L} \sum_{\beta=-\alpha}^{\alpha} 4 \pi(-1)^{\frac{\alpha}{2}} I_{n n^{\prime} \alpha}(q) Q_{l l^{\prime} \alpha}^{m m^{\prime} \beta} Y_{\alpha}^{\beta}(\mathbf{u}) \\
I_{n n^{\prime} \alpha}(q \mid \zeta)=\int_{0}^{\infty} G_{n}(R \mid \zeta) G_{n^{\prime}}(R \mid \zeta) j_{\alpha}(2 \pi q R) R^{2} \mathrm{~d} R, \quad Q_{l l^{\prime} \alpha}^{m m^{\prime} \beta}=\int_{\mathbb{S}^{2}} Y_{l}^{m}(\mathbf{r}) Y_{l^{\prime}}^{m^{\prime}}(\mathbf{r}) Y_{\alpha}^{\beta}(\mathbf{r}) \mathrm{d} \mathbf{r}
\end{gathered}
$$

The spherical integration $Q_{l l^{\prime} \alpha}^{m m^{\prime} \beta}$ is the integration of three SHs, which can be calculated by Wigner 3-j symbol. Please note that the summation over $\alpha$ in 5 is up to $2 L$, because if $\alpha>2 L$, then $\alpha>2 L \geq l+l^{\prime}$ violates the triangle inequality and $Q_{l l^{\prime} \alpha}^{m m^{\prime} \beta}=0$. The radial integration in Eq. (7) can be written in (8),

$$
I_{n n^{\prime} \alpha}(q \mid \zeta)=\kappa_{n}(\zeta) \kappa_{n^{\prime}}(\zeta) \frac{\zeta^{1.25}}{2 \sqrt{q}} \int_{0}^{\infty} x^{1.5} \exp \left(-x^{2}\right) L_{n}^{0.5}\left(x^{2}\right) L_{n^{\prime}}^{0.5}\left(x^{2}\right) J_{\alpha+0.5}(2 \pi q x \sqrt{\zeta}) \mathrm{d} x
$$

where $J_{\alpha+0.5}(x)=\sqrt{\frac{2 x}{\pi}} j_{\alpha}(x)$ is the Bessel function. Consider $L_{n}^{0.5}(x)=\sum_{i=0}^{n} l_{n}^{i} x^{i}, l_{n}^{i}=$ $(-1)^{i}\left(\begin{array}{c}n+0.5 \\ n-i\end{array}\right) \frac{1}{i !}$, then $L_{n}^{0.5}\left(x^{2}\right) L_{n^{\prime}}^{0.5}\left(x^{2}\right)=\sum_{i=0}^{n+n^{\prime}} h_{n n^{\prime}}^{i} x^{2 i}, h_{n n^{\prime}}^{i}=\sum_{j=0}^{\min (n, i)} l_{n}^{j} l_{n^{\prime}}^{i-j} \delta\left(i-j \leq n^{\prime}\right)$ Thus the radial integration can be solved based on the property of Bessel function [5],

$$
I_{n n^{\prime} \alpha}(q \mid \zeta)=\kappa_{n}(\zeta) \kappa_{n^{\prime}}(\zeta) \frac{\zeta^{0.5 \alpha+1.5} \pi^{\alpha+0.5} q^{\alpha}}{4 \Gamma(\alpha+1.5)} \sum_{i=0}^{n+n^{\prime}} h_{n n^{\prime}}^{i} \Gamma\left(\frac{1}{2} \alpha+i+\frac{3}{2}\right)_{1} F_{1}\left(\frac{2 i+\alpha+3}{2} ; \alpha+\frac{3}{2} ;-\pi^{2} q^{2} \zeta\right)
$$


where ${ }_{1} F_{1}$ is the confluent hypergeometric function. The final quadratic relation $E(\mathbf{q} \mid \boldsymbol{c})=$ $\boldsymbol{c}^{T} \mathbf{K}(\mathbf{q} \mid \zeta) \boldsymbol{c}$ is quite compact, where the kernel $\mathbf{K}(\mathbf{q} \mid \zeta)$ is a $N(L+1)(L+2) / 2$ dimensional symmetric matrix for each fixed $\mathbf{q}$ and $\zeta$, and $\mathbf{K}(\mathbf{q} \mid \zeta)$ is independent of data $E(\mathbf{q})$.

\subsection{Estimation of the Wavefunction Directly from the Signal}

With the analytical relation in (5), we propose to estimate the coefficient $c$ from the signal samples $\left\{E_{i}\right\}_{i=1}^{N_{s}}$ by minimizing the cost function $M(c)$ in (10), where the regularization matrix $\Lambda$ is the diagonal matrix with the elements $\Lambda_{n l m}=\lambda_{n} n^{2}(n+1)^{2}+\lambda_{l} l^{2}(l+1)^{2}$ which is motivated by QBI [7] and has been successfully used in SPFI [1 [5]3].

$$
\boldsymbol{c}=\underset{\|\boldsymbol{c}\|=1}{\arg \min } M(\boldsymbol{c}), \quad M(\boldsymbol{c})=\frac{1}{2} \sum_{i=1}^{N_{s}}\left(\boldsymbol{c}^{T} \mathbf{K}\left(\mathbf{q}_{i} \mid \zeta\right) \boldsymbol{c}-E_{i}\right)^{2}+\frac{1}{2} \boldsymbol{c}^{T} \Lambda \boldsymbol{c}
$$

The minimization must be performed in a high dimensional sphere because of the constraint $\|\boldsymbol{c}\|=1$. The Riemannian gradient $\nabla M(\boldsymbol{c})$ on the sphere is

$$
\nabla M(\boldsymbol{c})=\frac{\partial M(\boldsymbol{c})}{\partial \boldsymbol{c}}-\left(\boldsymbol{c}^{T} \frac{\partial M(\boldsymbol{c})}{\partial \boldsymbol{c}}\right) \boldsymbol{c}, \quad \frac{\partial M(\boldsymbol{c})}{\partial \boldsymbol{c}}=\sum_{i=1}^{N_{s}} 2\left(\boldsymbol{c}^{T} \mathbf{K}\left(\mathbf{q}_{i} \mid \zeta\right) \boldsymbol{c}-E_{i}\right) \mathbf{K}\left(\mathbf{q}_{i} \mid \zeta\right) \boldsymbol{c}+\Lambda \boldsymbol{c}
$$

Then we propose a gradient descent method in (12), where $c^{(k)}$ means $c$ in $k$-th step, $d t$ is the step size from the standard line search method, $\operatorname{Exp}_{c}(v)$ is the exponential map [6]. See Algorithm 1.1 for the details, where we initially set $c^{(0)}=(1,0, \ldots, 0)^{T}$ to represent a typical isotropic Gaussian EAP, and $d t_{0}=0.1$ experimentally, considering $\|\boldsymbol{c}\|=1$. Note that $\boldsymbol{c}^{(k)}$ in each step satisfies $\left\|\boldsymbol{c}^{(k)}\right\|=1$ thanks to the Riemannian framework [6].

$$
\boldsymbol{c}^{(k+1)}=\operatorname{Exp}_{\boldsymbol{c}^{(k)}}\left(-d t \frac{\nabla M\left(\boldsymbol{c}^{(k)}\right)}{\left\|\nabla M\left(\boldsymbol{c}^{(k)}\right)\right\|}\right), \quad \text { where } \quad \operatorname{Exp}_{\boldsymbol{c}}(\boldsymbol{v})=\boldsymbol{c} \cos \|\boldsymbol{v}\|+\frac{\boldsymbol{v}}{\|\boldsymbol{v}\|} \sin \|\boldsymbol{v}\|
$$

\subsection{Estimation of the Nonnegative Definite EAP and ODFs}

After the coefficient $c$ is estimated from signal samples $\left\{E_{i}\right\}$, the $\operatorname{EAP} P(\mathbf{R})=(\psi(\mathbf{R}))^{2}$ is naturally nonnegative definite in $\mathbb{R}^{3}$, and $\int_{\mathbb{R}^{3}} P(\mathbf{R}) \mathrm{d} \mathbf{R}=1$ because $\|\boldsymbol{c}\|=1$ is forced in each estimation step. For given radius $R_{0}$, the EAP profile can be represented by product of SH basis or SH basis, considering $Y_{l}^{m}(\mathbf{r}) Y_{l^{\prime}}^{m^{\prime}}(\mathbf{r})=\sum_{\alpha \beta} Y_{\alpha}^{\beta}(\mathbf{r}) Q_{l l^{\prime} \alpha}^{m m^{\prime} \alpha}$.

$$
P\left(R_{0} \mathbf{r}\right)=(\psi(\mathbf{R}))^{2}=\sum_{\alpha=0}^{2 L} \sum_{\beta=-\alpha}^{\alpha}\left(\sum_{n l m} \sum_{n^{\prime} l^{\prime} m^{\prime}} c_{n l m} c_{n^{\prime} l^{\prime} m^{\prime}} G_{n}\left(R_{0}\right) G_{n^{\prime}}\left(R_{0}\right) Q_{n n^{\prime} \alpha}^{m m^{\prime} \beta}\right) Y_{\alpha}^{\beta}(\mathbf{r})
$$

Two kinds of ODFs $\Phi_{0}(\mathbf{r})$ and $\Phi_{2}(\mathbf{r})$ are also nonnegative definite in $\mathbb{S}^{2}$ because they are radial integrations of nonnegative $P(\mathbf{R})$. The ODFs can be analytically represented by product of SH basis or SH basis with the estimated coefficient $c$. For $\Phi_{2}(\mathbf{r})$, we have

$$
\begin{aligned}
\Phi_{2}(\mathbf{r}) & =\int_{0}^{\infty}(\psi(\mathbf{R}))^{2} R^{2} \mathrm{~d} R=\sum_{n l m} \sum_{n^{\prime} l^{\prime} m^{\prime}}\left(\int_{0}^{\infty} G_{n}(R) G_{n^{\prime}}(R) R^{2} \mathrm{~d} R\right) c_{n l m} c_{n^{\prime} l^{\prime} m^{\prime}} Y_{l}^{m}(\mathbf{r}) Y_{l^{\prime}}^{m^{\prime}}(\mathbf{r}) \\
& =\sum_{n l m} \sum_{l^{\prime} m^{\prime}} c_{n l m} c_{n l^{\prime} m^{\prime}} Y_{l}^{m}(\mathbf{r}) Y_{l^{\prime}}^{m^{\prime}}(\mathbf{r})=\sum_{\alpha=0}^{2 L} \sum_{\beta=-\alpha}^{\alpha}\left(\sum_{n l m} \sum_{l^{\prime} m^{\prime}} c_{n l m} c_{n l^{\prime} m^{\prime}} Q_{l l^{\prime} \alpha}^{m m^{\prime} \beta}\right) Y_{\alpha}^{\beta}(\mathbf{r})
\end{aligned}
$$




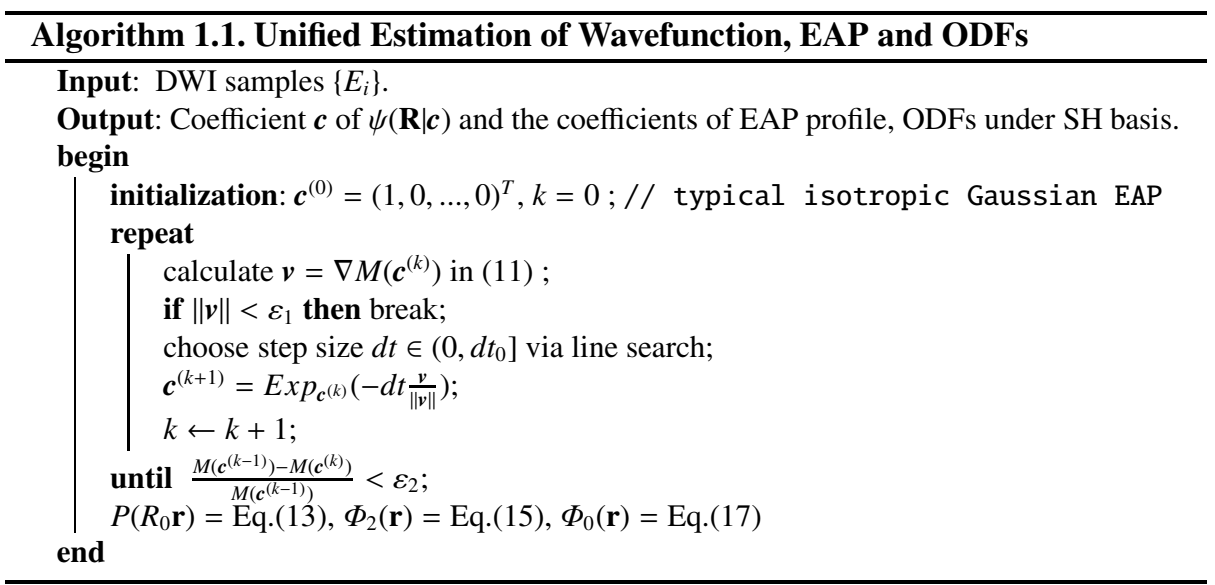

Here we use the orthogonality $\int_{0}^{\infty} G_{n}(R) G_{n^{\prime}}(R) R^{2} \mathrm{~d} R=\delta_{n n^{\prime}}$ [1]. It is clear in (15) that $\int_{\mathbb{S}^{2}} \Phi_{2}(\mathbf{r}) \mathrm{d} \mathbf{r}=\sum_{n l m} c_{n l m}^{2}=1$, because of the orthogonality of SHs. So the estimated $\Phi_{2}(\mathbf{r})$ from SRPE is indeed the nonnegative definite marginal EAP. For $\Phi_{0}(\mathbf{r})$, we have

$$
\begin{array}{r}
\Phi_{0}(\mathbf{r})=\frac{1}{Z} \int_{0}^{\infty}(\psi(\mathbf{R}))^{2} \mathrm{~d} R=\frac{1}{Z} \sum_{n l m} \sum_{n^{\prime} l^{\prime} m^{\prime}}\left(\int_{0}^{\infty} G_{n}(R) G_{n^{\prime}}(R) \mathrm{d} R\right) c_{n l m} c_{n^{\prime} l^{\prime} m^{\prime}} Y_{l}^{m}(\mathbf{r}) Y_{l^{\prime}}^{m^{\prime}}(\mathbf{r}) \\
\int_{0}^{\infty} G_{n}(R) G_{n^{\prime}}(R) \mathrm{d} R=\kappa_{n}(\zeta) \kappa_{n^{\prime}}(\zeta) \frac{\sqrt{\zeta}}{2} \int_{0}^{\infty} \exp (-x) \sum_{i=0}^{n+n^{\prime}} h_{n n^{\prime}}^{i} x^{i-0.5} \mathrm{~d} x=\kappa_{n}(\zeta) \kappa_{n^{\prime}}(\zeta) \frac{\sqrt{\zeta}}{2} \sum_{i=0}^{n+n^{\prime}} h_{n n^{\prime}}^{i} \Gamma\left(i+\frac{1}{2}\right)
\end{array}
$$

where $\Gamma(\cdot)$ is the Gamma function. Then $\Phi_{0}(\mathbf{r})$ is represented as

$$
\Phi_{0}(\mathbf{r})=\frac{\sqrt{\zeta}}{2 Z} \sum_{\alpha=0}^{2 L} \sum_{\beta=-\alpha}^{\alpha}\left(\sum_{n l m} \sum_{n^{\prime} l^{\prime} m^{\prime}} \kappa_{n}(\zeta) \kappa_{n^{\prime}}(\zeta) c_{n l m} c_{n^{\prime} l^{\prime} m^{\prime}} Q_{l l^{\prime} \alpha}^{m m^{\prime} \beta} \sum_{i=0}^{n+n^{\prime}} h_{n n^{\prime}}^{i} \Gamma\left(i+\frac{1}{2}\right)\right) Y_{\alpha}^{\beta}(\mathbf{r})
$$

\subsection{Implementation}

The implementation of SRPE has two steps. The first step is to estimate coefficient vector $c$ of $\psi(\mathbf{R})$ from signal samples $\left\{E_{i}\right\}$. The second step is to obtain EAP and ODFs analytically from formulae (13) (15) (17), which is independent of the first step. The whole estimation error is only in the first step, because the second step is analytical.

In SPFI, the artificial shell at $q=0$ needs to be considered for the prior $E(0)=1$ [5], which largely improves the results of SPFI. However, in SRPE $E(0)=1$ is naturally satisfied because $\|\boldsymbol{c}\|=1=\int_{\mathbb{R}^{3}} P(\mathbf{R}) \mathrm{d} \mathbf{R}=E(0)$ is forced in estimation, which can be seen as an advantage over SPFI, thanks to the Riemannian framework [6]. Similarly with SPFI, the scale $\zeta$ needs to be chosen in the first step. SPFI proposed to set $\zeta$ using a typical Apparent Diffusion Coefficient (ADC) value $D_{0}=0.7 \times 10^{-3} \mathrm{~mm}^{2} / \mathrm{s}[5]$. Motivated by SPFI, we propose to set $\zeta$ by two ways. The first way is to set $\zeta=4 \tau D_{0}$ such that the first SPF basis $G_{0}(R) Y_{0}^{0}(\mathbf{r}) \propto \exp \left(-\frac{R^{2}}{8 \tau D_{0}}\right)$ can represent typical isotropic signal $E(\mathbf{q})=\exp \left(-4 \pi^{2} \tau q^{2} D_{0}\right)$ with the typical isotropic Gaussian $\operatorname{EAP} N\left(\mathbf{R} / 2 \tau D_{0}\right) . \tau$ 
is the diffusion time to calculate $q$ from $b$ value, i.e. $b=4 \pi^{2} \tau q^{2}$. In this way $\zeta$ is shared by all voxels, thus the kernels $\left\{\mathbf{K}\left(\mathbf{q}_{i} \mid \zeta\right)\right\}_{i=1}^{N_{s}}$ need to be calculated only once on samples $\left\{\mathbf{q}_{i}\right\}$. However the typical ADC value $D_{0}$ may be not appropriate for the voxels with the $\mathrm{ADC}$ far from $D_{0}$. Thus the second way is to adaptively estimate the isotropic tensor with ADC $D_{i s o}$ from signal $\left\{E_{i}\right\}_{i=1}^{N_{s}}$ in each voxel, which can be done by a standard least square estimation in DTI. Then we set $\zeta=4 \tau D_{\text {iso }}$. The isotropic EAP $N\left(\mathbf{R} \mid 2 \tau D_{i s o}\right)$ may better approximate the signal samples than the EAP $N\left(\mathbf{R} \mid 2 \tau D_{0}\right)$ provided by $D_{0}$. In this way $\zeta$ is adaptively set for each voxel, thus the $\operatorname{kernel}\left\{\mathbf{K}\left(\mathbf{q}_{i} \mid \zeta\right)\right\}_{i=1}^{N_{s}}$ needs to be calculated for each voxel, which can be accelerated by reusing the pre-calculated $\left\{\mathbf{K}\left(\mathbf{q}_{i} \mid \zeta\right)\right\}_{i=1}^{N_{s}}$ with the close scale. Note that the adaptive $D_{i s o}$ can be also used in SPFI. The used special functions are implemented in GSL, which is very efficient. The computation burden is in the calculation of the kernel $\mathbf{K}$ and the summation in (11) for many times. With our $\mathrm{C}++$ codes in ordinary PC, for 10000 voxels, it takes about 7 minutes with fixed scale and 20 minutes with adaptive scale.

\section{Experiments}

In practice we found that the ODFs estimated by many HARDI methods normally have only a small number of negative values close to zero when the SNR is very low, however even with high SNR, the negative values are serious for the estimated EAPs especially for large radius $R$. Thus we focus on EAP estimation in experiments.

Synthetic Data. The synthetic data were generated from mixture of tensor model [7] where two tensors cross with a given angle in $\left[45^{\circ}, 90^{\circ}\right]$. Three shells $(b=500,1500,3000$ $\mathrm{s} / \mathrm{mm}^{2}$ ) were used, 60 samples per shell. EAP profiles with radius $R_{0}=15 \mu \mathrm{m}$ were estimated by SPFI and SRPE. The Normalized Mean Square Error (NMSE) between the ground truth EAP profile $P\left(R_{0} \mathbf{r}\right)$ and the estimated EAP profile $\widetilde{P}\left(R_{0} \mathbf{r}\right)$ is defined as $\frac{\sqrt{\int_{S^{2}}\left|\widetilde{P}\left(R_{0} \mathbf{r}\right)-P\left(R_{0} \mathbf{r}\right)\right|^{2} \mathrm{dr}}}{\sqrt{\int_{S^{2}} \mid P\left(R_{0} \mathbf{r}\right)^{2} \mathrm{dr}}}$. In the noise-free experiment, the signal was generated from two tensor configurations with eigenvalues $T_{1}=(1.7,0.3,0.3) \times 10^{-3} \mathrm{~mm}^{2} / \mathrm{s}$ and $T_{2}=$ $(0.9,0.3,0.3) \times 10^{-3} \mathrm{~mm}^{2} / \mathrm{s}$. We set $N=2, L=4, \lambda_{l}=\lambda_{n}=0$ and considered both typical scale and adaptive scale for SPFI and SRPE . Fig. 11(A2,A3) recorded the NMSE when two maxima were detected. SRPE generally obtains lower NMSE and has better angular resolution than SPFI. The adaptive scale obtains lower NMSE in two methods when $T_{2}$ is used, which is because the ADC in tensor $T_{1}$ is much close to the typical $D_{0}$, while the ADC in $T_{2}$ is not. Fig. 11A1) shows the ground truth EAP and the estimated EAPs by two methods when $T_{1}$ and crossing angle of $55^{\circ}$ are used. SRPE has better angular resolution and avoids the negative values around the original point in the EAP by SPFI. Note that the EAP profile estimated by SPFI in (A1) has more than $20 \%$ negative values showed in the blue square, although only $1 \%$ points are negative and have absolute values larger than one tenth of the maximal value of the EAP profile. In the experiment with Rician noise, $T_{1}$ and adaptive scale were used. We set $\lambda_{l}=\lambda_{n}=10^{-8}$ for SPFI suggested in [5]. Since the coefficient $c$ in SRPE has different range $(\|c\|=1)$ from coefficients in SPFI, in order to perform a fair comparison, we still set $\lambda_{l}=\lambda_{n}=0$ for SRPE without any regularization. The estimation was performed for 1000 trials with 

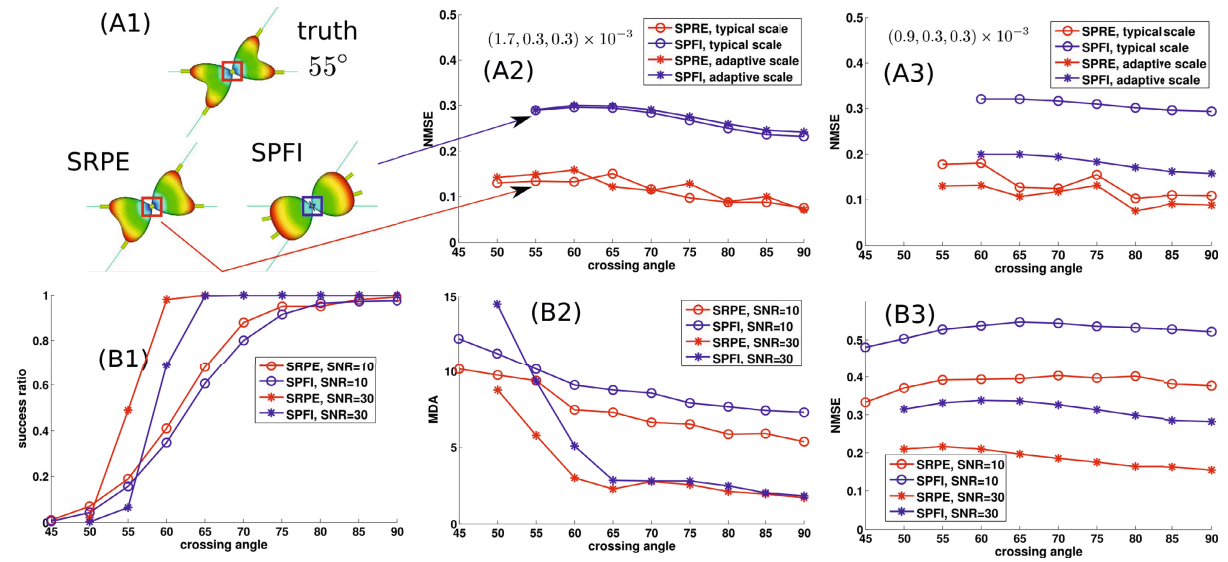

Fig. 1. A1: ground truth EAP and estimated EAPs from two methods, where the EAP by SPFI has negative values in the blue square. The long thin sticks and short thick sticks are the ground truth directions and the detected maxima respectively. A2, A3: NMSE in noise free experiment for two tensor configurations $T_{1}, T_{2}$. B1, B2, B3: success ratio, MDA and the mean of NMSE in the experiments with $S N R=10,30$.

$S N R=10,30$, where the success ratio was recored when two maxima were detected, the Mean Difference of Angle (MDA) was calculated in the successful trials, and the mean of NMSE was calculated over all trials. See Fig. 1 B1,B2,B3). It is clear that SRPE generally has higher success ratio, lower MDA and lower NMSE than SPFI.

Real Monkey Data. We perform SRPE and SPFI in a real monkey data with three $b$ values $\left(b=500,1500,3000 \mathrm{~s} / \mathrm{mm}^{2}\right), 30$ gradients per shell. $N=2, L=4 \lambda_{n}=\lambda_{l}=$ $10^{-8}$ were set for SPFI. In order to perform a fair comparison, we set $N=2, L=4$, $\lambda_{n}=\lambda_{l}=0$ for SRPE. See Fig. 2 for the estimated EAPs and ODFs in an enlarged area. The fifth column demonstrates that the ODFs $\Phi_{2}(\mathbf{r})$ by two methods are similar. It is probably because the estimated ODFs by SPFI in this area only have averagely $0.03 \%$

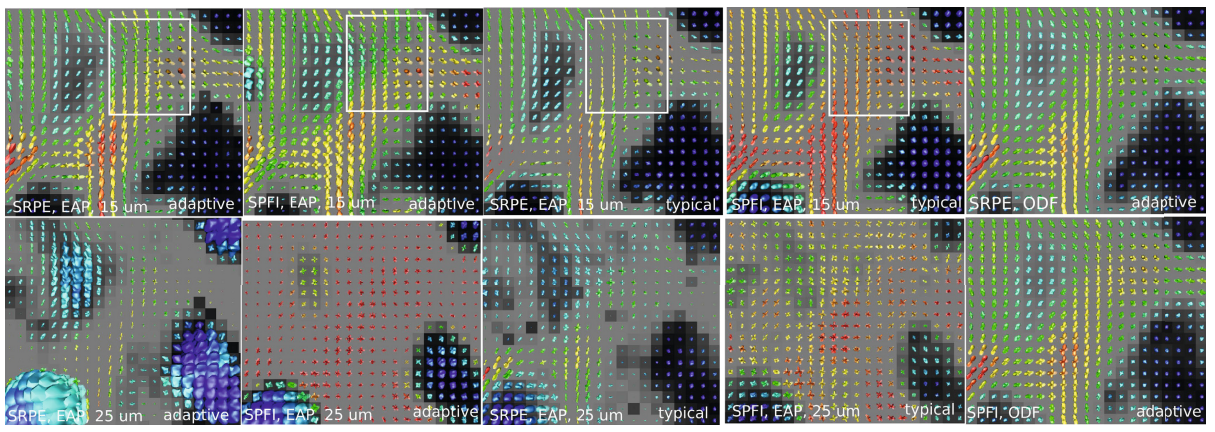

Fig. 2. The first four columns are the EAP profiles at 15, 25 $\mu \mathrm{m}$ estimated by SRPE and SPFI with adaptive and typical scales. The last column shows the ODF $\Phi_{2}(\mathbf{r})$ estimated by SRPE and SPFI with adaptive scale. The EAP profiles and ODFs are colored by generalized FA (GFA) [11]. 
negative values. The EAP profiles at radius $25 \mu \mathrm{m}$ and $15 \mu \mathrm{m}$ were estimated by two methods with both adaptive and typical scales. For both scale settings, the SPFI obtains very noisy EAP profiles at $25 \mu \mathrm{m}$, while SRPE obtains cleaner results. That's probably because the EAP profiles at $25 \mu \mathrm{m}$ by SPFI in this area have averagely more than $20 \%$ negative values, while the EAPs by SRPE are always nonnegative. Note that the EAP profiles by SRPE with adaptive scale is sharper than the EAPs obtained by typical scale, and both SPFI and SRPE obtain isotropic EAP profile in grey matter areas, which is an important advantage over other methods like QBI.

\section{Conclusion}

In this paper, we propose a unified model-free multi-shell HARDI method, named as SRPE, to estimate simultaneously the wavefunction of EAP, the nonnegative definite EAP and two kinds of ODFs from the diffusion signals. To the best of our knowledge, this is the first work on nonnegative definite EAP and ODFs estimation in the continuous domains, although some other methods have considered the nonnegative constraint on some given discrete samples. SRPE generalizes the positive definite tensor estimation based on the Riemannian framework for tensors to the nonnegative definite EAP and ODFs estimation by considering the Riemannian framework for EAPs. The experiments on synthetic data and real data demonstrated that the negative values happen in reconstruction methods like SPFI even without noise. This phenomenon is more serious for EAP profiles with larger radius. SRPE can improve the estimation results by avoiding the negative values, and it generally has better EAP estimation than SPFI especially for the EAP profile with large radius. The ODFs by SRPE and SPFI seem to be similar, probably because the estimated ODFs by most HARDI methods are so smooth that they seldom have negative values or have the negative values with small absolute values.

\section{References}

1. Assemlal, H.E., Tschumperlé, D., Brun, L.: Efficient and robust computation of PDF features from diffusion MR signal. Medical Image Analysis 13, 715-729 (2009)

2. Callaghan, P.T.: Principles of nuclear magnetic resonance microscopy. Oxford University Press (1991)

3. Cheng, J., Ghosh, A., Deriche, R., Jiang, T.: Model-Free, Regularized, Fast, and Robust Analytical Orientation Distribution Function Estimation. In: Jiang, T., Navab, N., Pluim, J.P.W., Viergever, M.A. (eds.) MICCAI 2010, Part I. LNCS, vol. 6361, pp. 648-656. Springer, Heidelberg (2010)

4. Cheng, J., Ghosh, A., Jiang, T., Deriche, R.: A Riemannian Framework for Orientation Distribution Function Computing. In: Yang, G.-Z., Hawkes, D., Rueckert, D., Noble, A., Taylor, C. (eds.) MICCAI 2009, Part I. LNCS, vol. 5761, pp. 911-918. Springer, Heidelberg (2009)

5. Cheng, J., Ghosh, A., Jiang, T., Deriche, R.: Model-Free and Analytical EAP Reconstruction via Spherical Polar Fourier Diffusion MRI. In: Jiang, T., Navab, N., Pluim, J.P.W., Viergever, M.A. (eds.) MICCAI 2010, Part I. LNCS, vol. 6361, pp. 590-597. Springer, Heidelberg (2010)

6. Cheng, J., Ghosh, A., Jiang, T., Deriche, R.: Diffeomorphism Invariant Riemannian Framework for Ensemble Average Propagator Computing. In: Fichtinger, G., Martel, A., Peters, T. (eds.) MICCAI 2011, Part II. LNCS, vol. 6892, pp. 98-106. Springer, Heidelberg (2011) 
7. Descoteaux, M., Angelino, E., Fitzgibbons, S., Deriche, R.: Regularized, Fast and Robust Analytical Q-ball Imaging. Magnetic Resonance in Medicine 58, 497-510 (2007)

8. Goh, A., Lenglet, C., Thompson, P.M., Vidal, R.: Estimating Orientation Distribution Functions with Probability Density Constraints and Spatial Regularity. In: Yang, G.-Z., Hawkes, D., Rueckert, D., Noble, A., Taylor, C. (eds.) MICCAI 2009, Part I. LNCS, vol. 5761, pp. 877-885. Springer, Heidelberg (2009)

9. Lenglet, C., Rousson, M., Deriche, R.: DTI segmentation by statistical surface evolution. IEEE Transactions on Medical Imaging 25, 685-700 (2006)

10. Özarslan, E., Koay, C., Shepherd, T., Blackband, S., Basser, P.: Simple harmonic oscillator based reconstruction and estimation for three-dimensional q-space mri. In: ISMRM (2009)

11. Tuch, D.S.: Q-ball imaging. Magnetic Resonance in Medicine 52, 1358-1372 (2004) 